\title{
Systematic review and meta-analysis of the diagnostic value of radionuclide imaging for thyroid nodules
}

\author{
Xin Song ${ }^{1,2 \#}$, Zhongmei Luo ${ }^{2,3 \#}$, Haiyan Sun ${ }^{2,3}$, Lingna Hao ${ }^{1,2}$ \\ ${ }^{1}$ Specialty of Geriatric Endocrinology, Sichuan Provincial People's Hospital, University of Electronic Science and Technology of China, Chengdu, \\ China; ${ }^{2}$ Chinese Academy of Sciences Sichuan Translational Medicine Research Hospital, Chengdu, China; ${ }^{3}$ Geriatric ICU, Sichuan Provincial \\ People's Hospital, University of Electronic Science and Technology of China, Chengdu, China \\ Contributions: (I) Conception and design: X Song, Z Luo; (II) Administrative support: H Sun; (III) Provision of study materials or patients: X Song, H \\ Sun, L Hao; (IV) Collection and assembly of data: All authors; (V) Data analysis and interpretation: All authors; (VI) Manuscript writing: All authors; \\ (VII) Final approval of manuscript: All authors. \\ "These authors contributed equally to this work. \\ Correspondence to: Lingna Hao; Haiyan Sun. Sichuan Provincial People's Hospital, University of Electronic Science and Technology of China, No. 32, \\ West 2, Section 1 Ring Road, Qingyang District, Chengdu 610072, China. Email: luckynurse1@163.com; Haiyan13982098651@163.com.
}

\begin{abstract}
Background: There are many studies on diagnostic imaging of thyroid nodules, among which radionuclide scanning imaging has been proven to be very effective in identifying malignant lesions of thyroid nodules and is now widely used in the clinical detection of thyroid nodules.

Methods: Studies on radionuclide imaging in the diagnosis of thyroid nodules were searched in multiple Chinese and English databases, including PubMed, Web of Science, Embase, and The Cochrane Library as of July 1, 2021. The search terms included "clinical experiment", "thyroid nodule", "radionuclide imaging", "thyroid mass", "thyroid masses", "postoperative pathology", and "histopathology". The basic information and evaluation indicators in the literature were screened and extracted. Subsequently, meta-analysis was performed using RevMan 5.3 and Stata 13 software provided by the Cochrane system. In addition, forest plots and summary receiver operating characteristic (SROC) curves were drawn.

Results: Seven randomized controlled trails (RCTs) were included in this study. The radionuclide imaging of thyroid nodule showed the area under SROC curve of 0.38 . The combined diagnostic sensitivity and specificity were 0.66 [95\% confidence interval (CI): 0.46, 0.89] and 0.36 (95\% CI: 0.21, 0.59), respectively. The combined positive likelihood ratio (LR) and negative LR were 0.66 (95\% CI: $0.53,0.87)$ and 0.67 (95\% CI: 0.43, 0.95), respectively. In addition, the combined diagnostic odds ratio (OR) was 4.45 (95\% CI: 0.25, 10.57).

Discussion: In this study, meta-analysis was used to confirm the application value of radionuclide imaging in the diagnosis of thyroid nodules. In the future, the value of radionuclide imaging in the diagnosis of thyroid nodules should be compared with other imaging detection methods.
\end{abstract}

Keywords: Radionuclide imaging; thyroid nodules; diagnosis; meta-analysis

Submitted Oct 27, 2021. Accepted for publication Dec 15, 2021.

doi: $10.21037 / g s-21-766$

View this article at: https://dx.doi.org/10.21037/gs-21-766

\section{Introduction}

Thyroid nodule refers to a mass in the thyroid gland, which can move up and down with the thyroid gland following the swallowing action. It is a common clinical disease and is caused by a variety of factors (1). Clinically, there are multiple thyroid diseases, such as thyroid degeneration, inflammation, autoimmunity, and new organisms, all of which can show nodules (2). Thyroid nodules can be solitary or multiple; the incidence of multiple nodules is higher than that of single nodules, but the incidence of single nodules 
is higher in thyroid cancer (3). Common clinical symptoms include pain around the nodules, pain in the throat, edema in the neck, and corresponding compression symptoms in the surrounding tissues of nodular compression (4). The known causes of thyroid nodules include normal thyroid hyperplasia, degeneration, heredity, and inflammation $(5,6)$.

Radionuclide scanning is one of the main adjuncts recommended in the latest ATA guidelines for the diagnosis and treatment of thyroid cancer [2009] (7). This modality displays the differences in the uptake of radiopharmaceuticals in various organs and lesioned tissues after they enter the body by imaging instruments (8). According to the imaging results, radionuclide scans can be divided into hot zone and cold zone imaging according to the uptake of radionuclides at the lesion site compared to normal tissue. And according to the imaging mode, it can be divided into static and dynamic imaging. Among them, dynamic imaging has obvious advantages due to the ability to display biochemical, functional, blood flow and morphological information together, and is often used for early diagnosis and efficacy observation of many diseases (9).

At present, the commonly used radionuclide imaging instruments include a scanner, Y camera, and emission computed tomography (ECT) camera. At present, ECT is more commonly used in clinical practice due to its good diagnostic effect. ECT is further divided into two types: positron type (PECT) and single photon type (SPECT). Among them, PETCT is a fusion of functional and anatomical image examination. SPECT works by using short half-life nuclide $99 \mathrm{mTc}$ and other labeled certain special compounds injected into the human body through a vein, $99 \mathrm{mTc}$ can emit $\gamma$-rays through decay, $\gamma$-rays will be converted into electrical signals and input into the computer, and reconstructed by computer tomography into a cross-sectional or three-dimensional image reflecting the physiological condition of a human organ. SPECT can either become a flat image or rotate around the body to acquire and reconstruct into a high-resolution tomographic image. Both PETCT and SPECT can provide two-dimensional static and dynamic images of organs or lesions, as well as three-dimensional images or optional tomographic images. can show deep tissue and lesions for true quantitative analysis (10). The main difference between these two is that PETCT can examine tumors sensitively and examine lesions at the molecular level whereas SPECT can only provide images and cannot determine the nature of the site examined. In addition, PETCT is a whole body examination, while SPECT can only examine local organs.
In recent years, radionuclide imaging has been widely and successfully used in the detection and characterization of various swelling, pain, and metastases. It is the main component of nuclear medicine, and the early diagnosis of thyroid nodules is one of its applications.

Currently, there are many studies on the diagnostic value of various imaging methods for thyroid nodules, but no uniform conclusion has been reached on the diagnostic value of radionuclide imaging in the clinical setting of thyroid nodules, and no systematic evaluation and metaanalysis studies specifically on ECT radionuclide imaging have appeared. Therefore, this study aims to analyze and summarize the relevant studies on the diagnosis of thyroid nodules by ECT radionuclide imaging published in China and abroad through systematic evaluation and meta-analysis, and then comprehensively evaluate the value of radionuclide imaging in the diagnosis of thyroid nodules, with the aim of providing scientific evidence to optimize the clinical diagnosis of thyroid nodules. We present the following article in accordance with the PRISMA reporting checklist (available at https://dx.doi.org/10.21037/gs-21-766).

\section{Methods}

\section{Literature search}

The China National Knowledge Internet (CNKI) database (1979-2021.4), China Biomedical Literature Database (1994-2021.4), Cochrane Library (2005-2021.4), Medline (1948-2021.4), PubMed (1978-2021.4), Web of Science (1984-2021.4) and Embase (1966.1-2021.4) were searched through computer. Published radionuclide imaging for the diagnosis of thyroid nodules related to randomized controlled trials were screened, and the relevant literature in the journal was manually searched. The compound Boolean logic retrieval method was adopted to select related documents. The Chinese databases was searched using a combination of the following search terms: "clinical experiments", "thyroid nodules", "radionuclide imaging", "thyroid mass", "thyroid masses", "postoperative pathology", and "histopathology". As for the English databases, the following search terms were applied: "clinical laboratory", "thyroid nodules", "radionuclide imaging", "thyroid masses", "Occupational thyroid lesions", "Postoperative pathology", and "Histopathology". All diagnostic laboratory studies were collected, and the research object was required to be human. The deadline for literature search was July 1, 2021. Subsequently, meta- 
analysis was performed using RevMan 5.3 and Stata 13 software provided by the Cochrane system.

The above search terms were combined freely, and the initial screening of the initially retrieved literatures was carried out by reading the titles and abstracts, so as to exclude inconsistent articles and identify studies that were eligible for inclusion. The second screening was conducted according to inclusion/exclusion criteria, and search engines were used to trace the included literature. The full texts of the included studies were read, and a third screening was conducted to evaluate the quality of the articles.

\section{Literature inclusion and exclusion criteria}

The inclusion criteria were as follows: (I) the types of diagnostic tests for radionuclide scanning of thyroid nodules included in all studies included prospective, retrospective, and cross-sectional studies; (II) studies involving patients with thyroid nodules that met the diagnostic criteria of a thyroid nodule; (III) radionuclide imaging was the standard to be measured, and postoperative histopathological results were used as the gold standard; (IV) articles with complete original data, and the number of true positive (TP), false positive (FP), true negative (TN), and false negative ( $\mathrm{FN}$ ) patients could be calculated directly or indirectly; and (V) articles with observational indicators such as sensitivity and specificity, which could be calculated indirectly.

The exclusion criteria were as follows: (I) reviews, conference papers, dissertations, newsletters, case reports, etc.; (II) second publications or repeated publication data; and (III) studies involving no direct or indirect extraction of TP, FP, TN, and FN data.

\section{Data extraction}

In this study, a special data extraction table was designed according to the research theme. Two experts used Microsoft Excel developer: Microsoft Corporation, whose headquarter is located in Redmond (Washington, USA) to independently extract data. After that, cross-checking was carried out. If the two experts had inconsistent views, a consistent conclusion was obtained through discussion. The following data was extracted from the included studies: (I) basic information of the included studies, such as first author, study title, study publication time, source of original literature, sample size of thyroid nodules patients, and mean age of patients; and (II) total number of benign and malignant thyroid nodules diagnosed by radionuclide scanning and postoperative histopathology, and the number of TP, FP, TN, and FN cases diagnosed by radionuclide scanning under the gold standard.

\section{Risk of bias and quality assessment}

Two investigators used RevMan 5.3 and the Diagnostic Test Accuracy Assessment Tool (QUADAS) to assess the quality of the included literature and the risk of bias. The literature with high indicators of heterogeneity was screened out individually and re-compared in a comprehensive way to assess the heterogeneity and sensitivity of the included literature. In the case of disagreement between investigators, a third investigator intervened in the evaluation and a consensus was eventually reached through discussion. The evaluation criteria included case selection, the method being evaluated, the gold standard, and the evaluation process and progress.

The QUADAS tool was used to evaluate the included literature when there were 16 items in total. "Yes", "No", and "Unclear" were adopted for literature quality assessment. "Yes" indicated that the standard was met, while "No" indicated that the standard was not met. Literature quality was defined as "Unclear" when information was incomplete or only partially met the criteria. Literature quality evaluation using RevMan 5.3 required quality evaluation in terms of the following aspects: (I) whether it was a randomized controlled study; (II) whether there was allocation concealment; (III) whether blind testing was applied; (IV) whether the result data was complete; (V) whether there was selective reporting; and (VI) whether there were other biases.

\section{Statistical analysis}

RevMan 5.3 was used to analyze the risk of bias of the included literature. The summary receiver operating characteristic (SROC) curve was used for diagnostic analysis. Consistency in the model was determined when the SROC curve presented a shoulder wall distribution, or the sensitivity and specificity were negatively correlated, and $\mathrm{P}<0.05$. Analysis of variance was used to test the consistency of the results, and the test level was set as $\alpha=0.1$. Therefore, when $\mathrm{I}^{2}<50 \%$ and $\mathrm{P}>0.05$, it was considered that there was no heterogeneity between the studies, so the fixed effect model was used for statistical analysis. However, when $\mathrm{I}^{2}>50 \%$ and $\mathrm{P}<0.05$, heterogeneity was considered to exist between the studies, and thus, the random effects 
Identification of studies via databases and registers

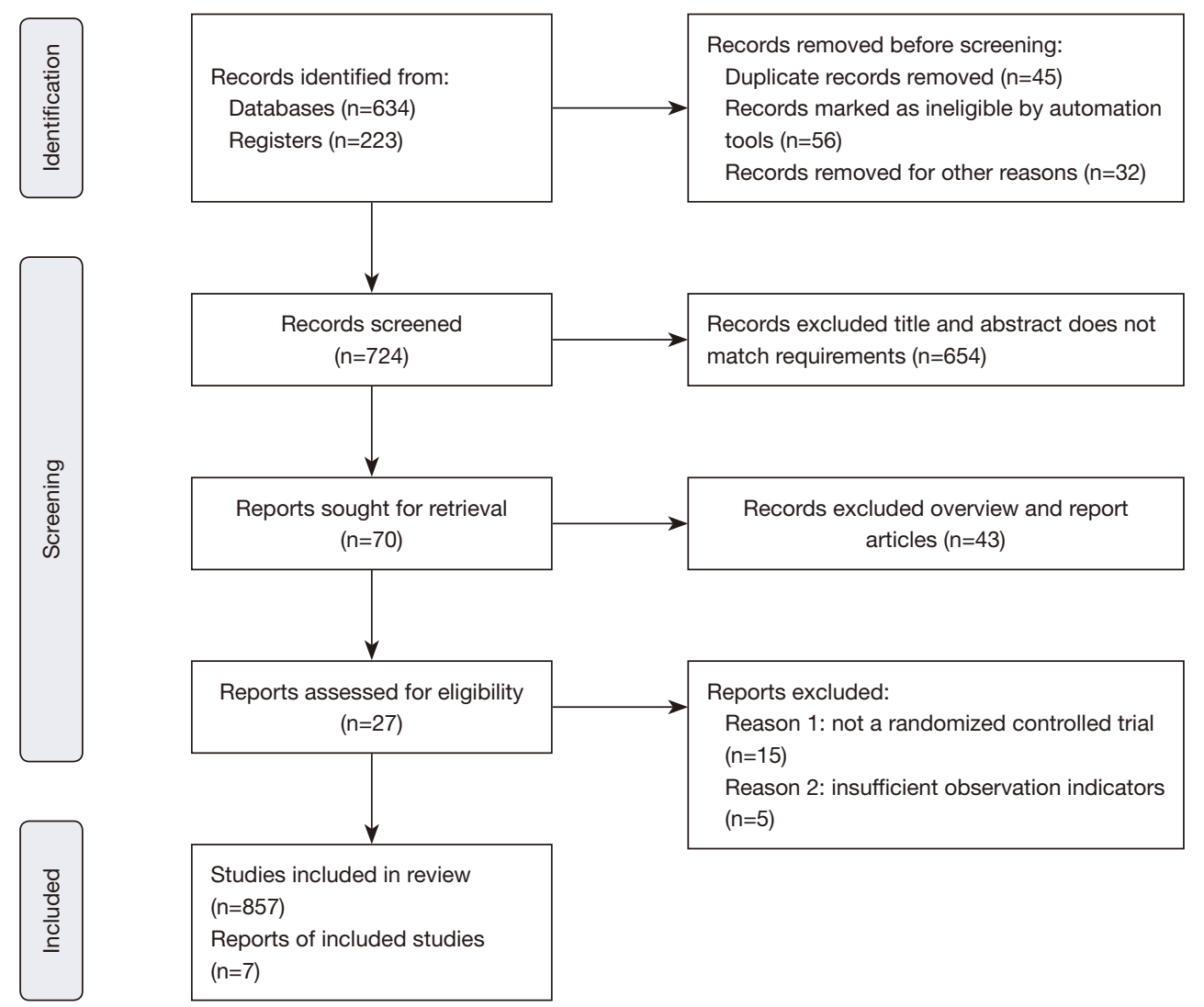

Figure 1 Literature retrieval flowchart.

model (REM) was used for statistical analysis. After construction of the corresponding SROC curve, the area under the SROC curve (AUC) was calculated; according to the AUC value of the diagnostic tests, an AUC of 0.5-0.7 was a considered to indicate a low diagnostic rate, an AUC of $0.7-0.9$ was considered moderate, and an AUC of 0.9-1.0 was considered high.

\section{Results}

\section{Search results and basic information of included documents}

A total of 857 articles were obtained initially, and 724 remained after exclusion. A total of 654 articles were excluded due to inconsistent titles and abstracts, 43 articles were excluded due to reviews and research reports, so 27 articles were remained. After 15 articles which were not RCTs and 5 articles with incomplete observation indicators were excluded, 7 articles were included finally (Figure 1).

A total of 1,152 patients were included in the 7 articles (11-17) meeting the inclusion criteria. The sample sizes of these 7 articles ranged from 44 to 496 , and the average age of included research objects was $42.23 \pm 7.84$ years old. All the 7 articles described the sample size, average age of patients, and the number of TP, FP, TN, and FN cases diagnosed by radionuclide scanning with postoperative pathological results as the gold standard in detail. The above information was summarized as Table 1 .

\section{Risk bias evaluation results of included studies}

Figures 2,3 display the multiple risk bias evaluation results of the included literature, as drawn by RevMan 5.3. In the seven randomized controlled trials, the degree of bias risk in all included articles was low. Overall, the number of "low risk" and "low concerns" in the included studies was 
Table 1 Basic characteristics of the included literatures

\begin{tabular}{|c|c|c|c|c|c|c|c|}
\hline First author & Year & Mean age of patients & Sample size & \multicolumn{4}{|c|}{ ECT } \\
\hline Basharat & $2011(11)$ & $33.04 \pm 12.29$ & 50 & 4 & 36 & 9 & 1 \\
\hline Deandreis & $2012(12)$ & 50.7 & 44 & 13 & 21 & 2 & 8 \\
\hline Lin & 2009 (13) & 43.5 & 326 & 48 & 195 & 66 & 17 \\
\hline Muñoz Pérez & $2013(15)$ & 47.5 & 46 & 17 & 12 & 1 & 16 \\
\hline Piccardo & 2016 (16) & $57 \pm 13$ & 87 & 10 & 32 & 8 & 37 \\
\hline Riazi & $2014(17)$ & $40.76 \pm 11.40$ & 104 & 9 & 20 & 0 & 74 \\
\hline
\end{tabular}

ECT, emission computed tomography; TN, true negative; TP, true positive; FN, false negative; FP, false positive.

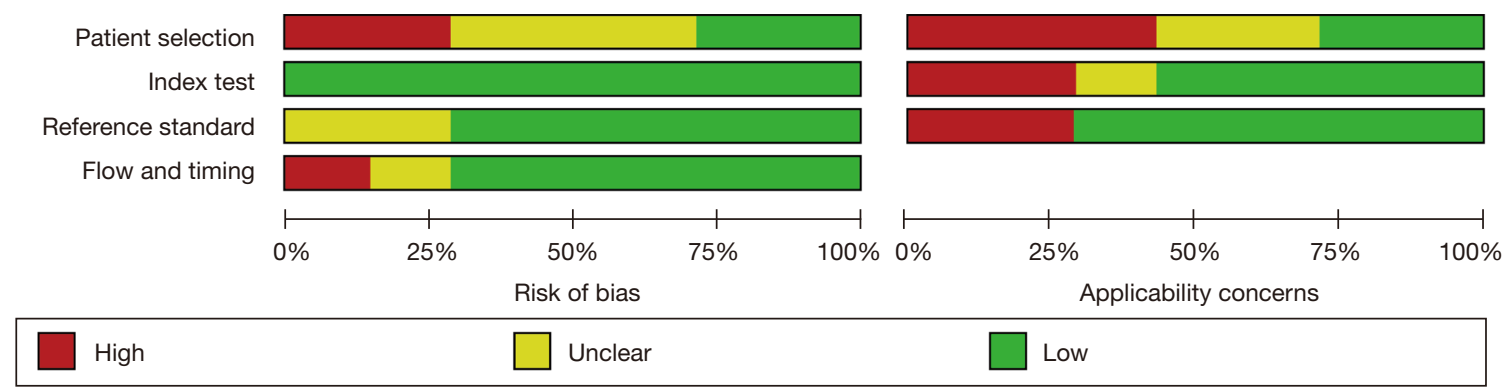

Figure 2 Risk of bias evaluation results of the included literature.

the largest, indicating that the included studies met the requirements of the analysis.

The QUADAS tool was used to evaluate the quality of each included article, and the results are shown in Table 2. All seven articles included in this study exhibited a low risk of bias and met the requirements of subsequent analysis.

\section{Meta-analysis of radionuclide imaging in the diagnosis of thyroid nodules}

Figure 4 was a forest map obtained by radionuclide scanning and imaging diagnosis of thyroid nodules. It illustrated that the estimated sensitivity range of ultrasound elastography in the diagnosis of pediatric surgical biliary atresia was $0.31-$ 1.00 , while the specificity of diagnosis was $0.01-1.00$.

The diagnosis of thyroid nodules using ECT was used to further systematically analyze the diagnosis results of 1,152 cases in 7 articles. The differences between the radionuclide imaging diagnosis of thyroid nodules and the real situation were summarized, and the obtained diagnostic sensitivity, specificity, positive likelihood ratio (LR), negative LR, and diagnostic ratio of radionuclide imaging were shown in Tables 3-7, respectively. The combined sensitivity showed Chi-square $=55.16$, degrees of freedom $(\mathrm{df})=6(\mathrm{P}<0.00000001)$, and inconsistency $(1$-square $)$ $=93.4 \%$, indicating the sensitivity of radionuclide scanning imaging to diagnose thyroid nodules was heterogeneity. The combined specificity of radionuclide imaging showed Chisquare $=265.24, \mathrm{df}=6(\mathrm{P}<0.00000001)$, and inconsistency $(1-$ square $)=97.7 \%$, showing heterogeneity. Combined positive LR showed Cochran-Q $=14.01, \mathrm{df}=6(\mathrm{P}<0.0062)$, inconsistency $(1$-square $)=72.6 \%$, and Tau-squared $=0.0328$, so there was heterogeneity. Combined negative LR showed Cochran- $\mathrm{Q}=12.01, \mathrm{df}=6(\mathrm{P}<0.0000001)$, inconsistency $(1$-square $)=68.4 \%$, and Tau-squared $=0.2031$, so there was heterogeneity. The combined diagnostic OR showed Cochran- $\mathrm{Q}=13.16, \mathrm{df}=6(\mathrm{P}<0.0000001)$, inconsistency $(1$-square $)=82.1 \%$, and Tau-squared $=0.4625$, so heterogeneity was obvious.

Since $\mathrm{P}<0.05$ and $\mathrm{I}^{2}>50 \%$, the heterogeneity was high, and REM was used for summary analysis. Finally, the combined sensitivity of the diagnosis of thyroid 


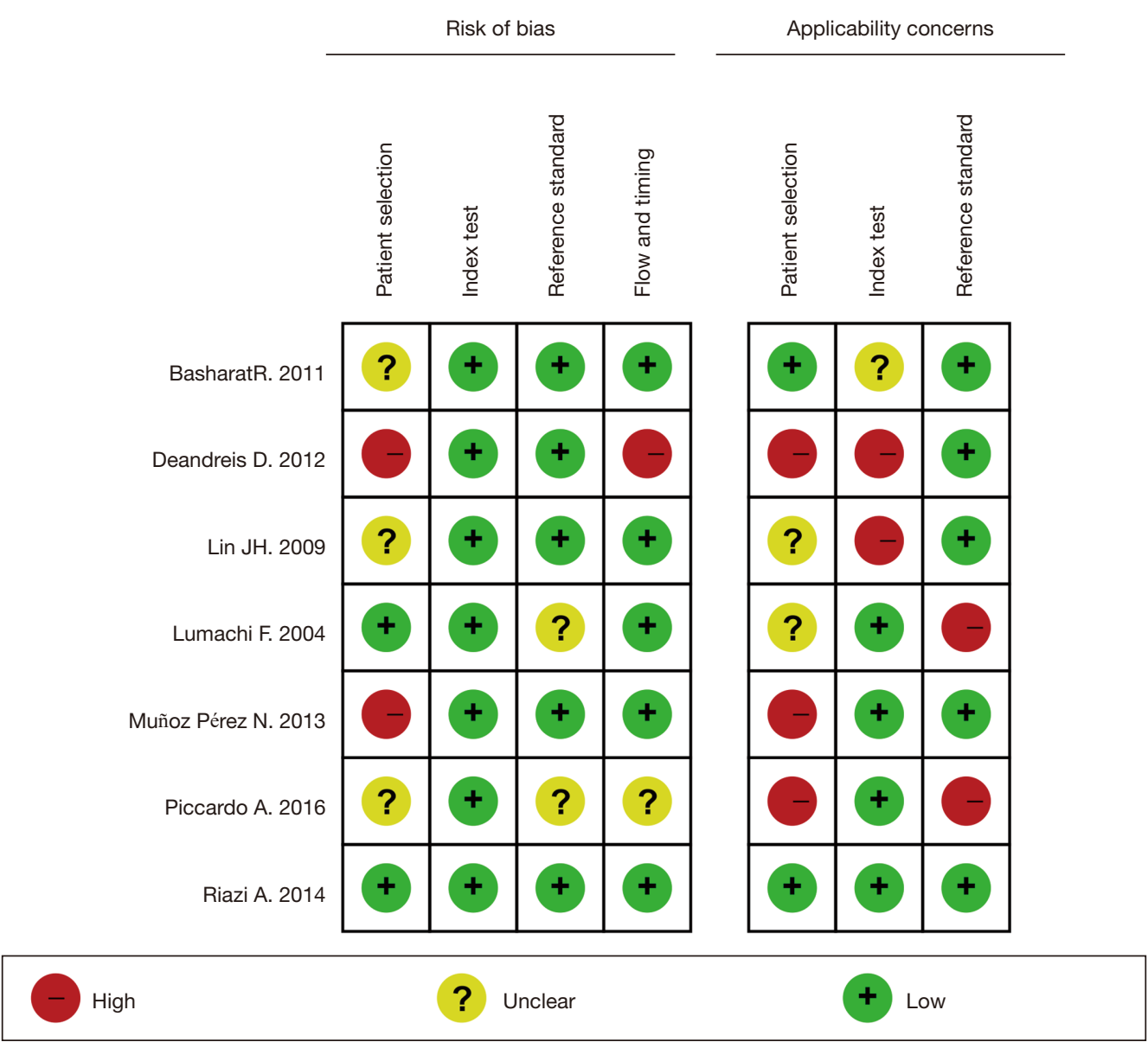

Figure 3 Multiple risk bias evaluation results corresponding to the multiple included studies. “+” signifies low risk of bias; “-” denotes high risk of bias; "?" indicates unclear risk of bias.

Table 2 Quality scores of included studies

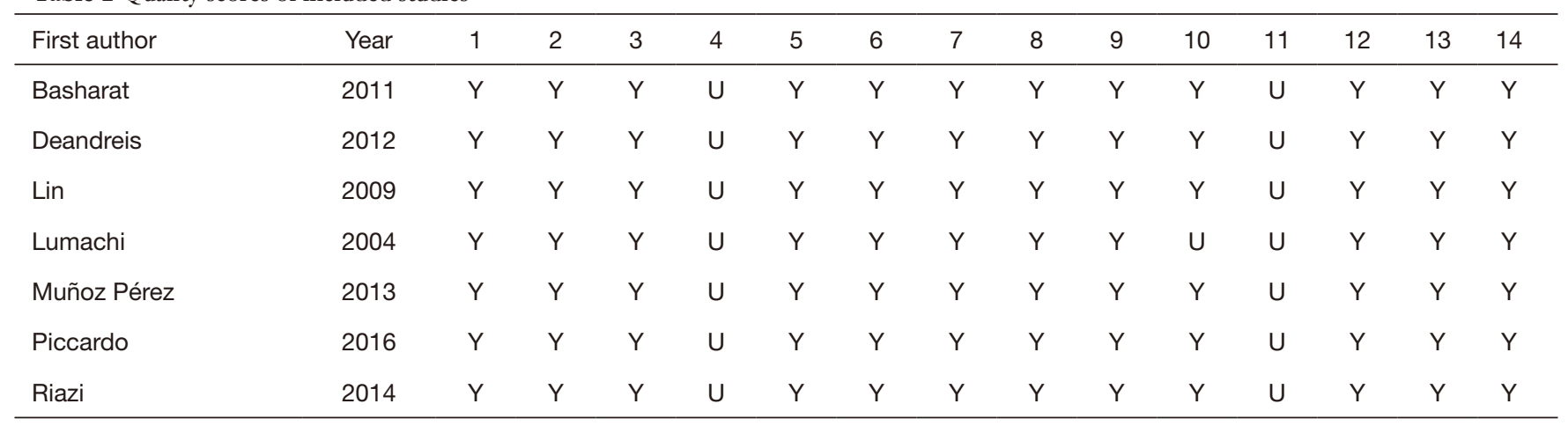

"1-14" showed the QUADAS entry standards; "Y" signifies yes; "U" denotes unclear; "N" indicates no. 


Study
Basharat R. 2011
Deandreis D. 2012
Lin JH. 2009
Lumachi F. 2004
Muñoz Pérez N. 2013
Piccardo A. 2016
Riazi A. 2014

$\begin{array}{rrrr}\text { TP } & \text { FP } & \text { FN } & \text { TN } \\ 4 & 36 & 9 & 1 \\ 13 & 21 & 2 & 8 \\ 48 & 195 & 66 & 17 \\ 92 & 315 & 85 & 4 \\ 17 & 12 & 1 & 16 \\ 10 & 32 & 8 & 37 \\ 9 & 20 & 0 & 74\end{array}$

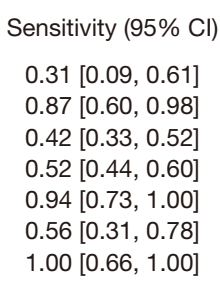

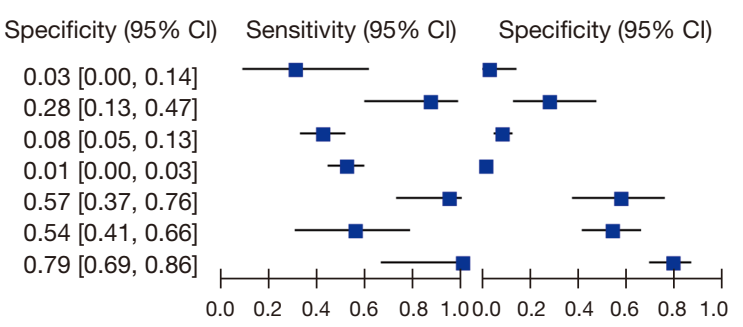

Figure 4 The forest map of thyroid nodules diagnosed by radionuclide imaging. TP, true positive; FP, false positive; FN, false negative; TN, true negative; CI, confidence interval.

Table 3 Diagnostic combined sensitivity of ECT in the included studies

\begin{tabular}{lc}
\hline Article & Sensitivity $(95 \% \mathrm{Cl})$ \\
\hline Basharat, 2011 & $0.31(0.09,0.61)$ \\
Deandreis, 2012 & $0.87(0.60,0.98)$ \\
Lin, 2009 & $0.42(0.33,0.52)$ \\
Lumachi, 2004 & $0.52(0.44,0.60)$ \\
Muñoz Pérez, 2013 & $0.94(0.73,1.00)$ \\
Piccardo, 2016 & $0.56(0.31,0.78)$ \\
Riazi, 2014 & $1.00(0.66,1.00)$ \\
\hline
\end{tabular}

ECT combined sensitivity $=0.66(0.46,0.89)$. Chi-square $=55.16$, $\mathrm{df}=6(\mathrm{P}<0.00000001)$. Inconsistency $(1$-square $)=93.4 \%$. ECT, emission computed tomography; $\mathrm{Cl}$, confidence interval; $\mathrm{df}$, degrees of freedom.

Table 4 Diagnostic specificity of ECT in the included studies

\begin{tabular}{lc}
\hline Article & Specificity $(95 \% \mathrm{Cl})$ \\
\hline Basharat, 2011 & $0.03(0.00,0.14)$ \\
Deandreis, 2012 & $0.28(0.13,0.47)$ \\
Lin, 2009 & $0.08(0.05,0.13)$ \\
Lumachi, 2004 & $0.01(0.00,0.03)$ \\
Muñoz Pérez, 2013 & $0.57(0.37,0.76)$ \\
Piccardo, 2016 & $0.54(0.41,0.66)$ \\
Riazi, 2014 & $0.79(0.69,0.86)$ \\
\hline
\end{tabular}

ECT combined specificity $=0.36(0.21,0.59)$. Chi-square $=265.24, \mathrm{df}=6(\mathrm{P}<0.00000001)$. Inconsistency (1-square) $=97.7 \%$. ECT, emission computed tomography; $\mathrm{Cl}$, confidence interval; df, degrees of freedom.
Table 5 Positive LRs of ECT diagnoses in the included studies

\begin{tabular}{lc}
\hline Article & Positive LRs \\
\hline Basharat, 2011 & 0.31 \\
Deandreis, 2012 & 0.87 \\
Lin, 2009 & 0.42 \\
Lumachi, 2004 & 0.52 \\
Muñoz Pérez, 2013 & 0.94 \\
Piccardo, 2016 & 0.56 \\
Riazi, 2014 & 1.00 \\
\hline
\end{tabular}

ECT combined positive LR $=0.66(0.53,0.87)$. Cochran-Q $=14.01, \mathrm{df}=6(\mathrm{P}<0.0062)$. Inconsistency $(1$-square $)=72.6 \%$, Tausquared $=0.0128$. LR, likelihood ratio; ECT, emission computed tomography; $\mathrm{Cl}$, confidence interval; df, degrees of freedom.

Table 6 Negative LRs of ECT diagnoses in the included studies

\begin{tabular}{lc}
\hline Article & Negative LRs \\
\hline Basharat, 2011 & 0.97 \\
Deandreis, 2012 & 0.72 \\
Lin, 2009 & 0.92 \\
Lumachi, 2004 & 0.99 \\
Muñoz Pérez, 2013 & 0.43 \\
Piccardo, 2016 & 0.46 \\
Riazi, 2014 & 0.21 \\
\hline
\end{tabular}

ECT combined negative LR $=0.67$ (0.43, 0.95). Cochran-Q $=12.01, \mathrm{df}=6(\mathrm{P}<0.0000001)$. Inconsistency $(1$-square $)=68.4 \%$, Tau-squared $=0.2031$. LR, likelihood ratio; ECT, emission computed tomography; $\mathrm{Cl}$, confidence interval; df, degrees of freedom. 
Table 7 OR of combined diagnosis of ECT diagnosis in the included studies

\begin{tabular}{lc}
\hline Article & OR of combined diagnosis \\
\hline Basharat, 2011 & 0.01 \\
Deandreis, 2012 & 2.48 \\
Lin, 2009 & 0.06 \\
Lumachi, 2004 & 0.01 \\
Muñoz Pérez, 2013 & 22.67 \\
Piccardo, 2016 & 1.45 \\
Riazi, 2014 & - \\
\hline
\end{tabular}

ECT combined diagnostic OR $=4.45(0.25,10.57)$. Cochran- $Q$ $=13.16, \mathrm{df}=6(\mathrm{P}<0.0000001)$. Inconsistency $(1$-square $)=82.1 \%$, Tau-squared $=0.4625$. OR, odds ratio; ECT, emission computed tomography; $\mathrm{Cl}$, confidence interval; df, degrees of freedom.

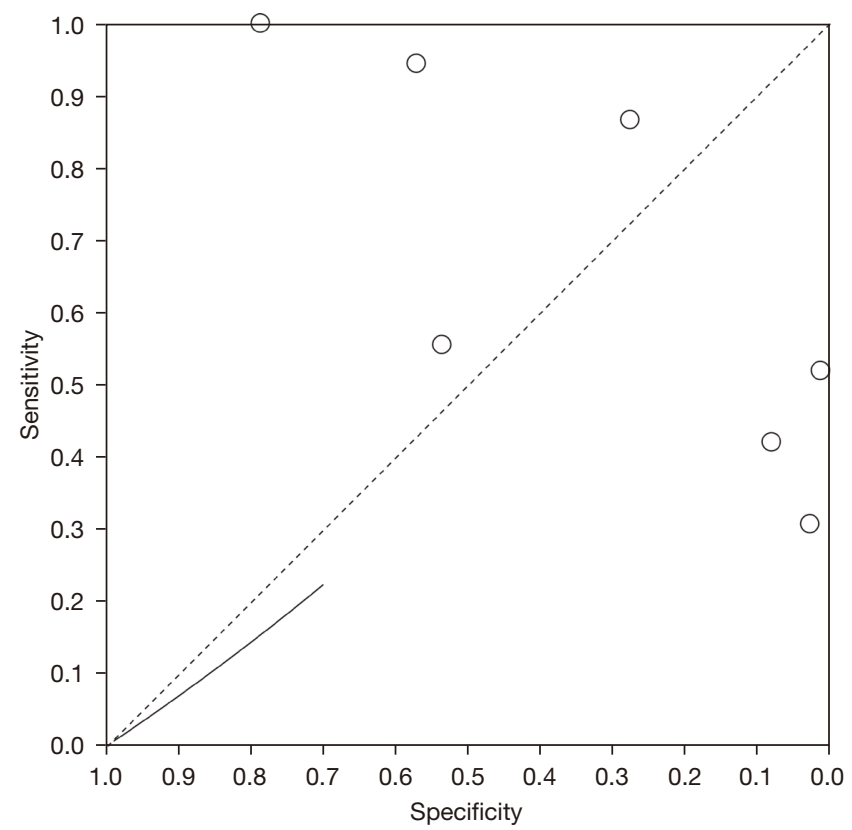

Figure 5 SROC curve of radionuclide imaging in the diagnosis of thyroid nodules. SROC, summary receiver operating characteristic.

nodules with radionuclide imaging was 0.66 , and the $95 \%$ confidence interval (CI) range was $(0.46,0.89)$. The combined specificity was 0.36 , and its $95 \%$ CI was $(0.21$, 0.59 ); the combined positive LR was 0.66 , and its $95 \%$ CI was $(0.53,0.87)$; the combined negative LR was 0.67 , and its $95 \%$ CI was $(0.43,0.95)$; and the combined diagnostic odds ratio (OR) was 4.45 , and its $95 \%$ CI was $(0.25,10.57)$.

\section{Radionuclide imaging diagnosis of SROC curve of thyroid nodules}

A bivariate model was used to analyze the value of radionuclide imaging in the diagnosis of thyroid nodules, and the results were shown in Figure 5. The aggregate estimate of sensitivity for different studies was $0.66(0.46$, 0.89 ), and the aggregate estimate of specificity was 0.36 (95\% CI: $0.21,0.59$ ). The AUC was 0.38 .

\section{Sensitivity analysis}

The results obtained from the meta-analysis after deleting the diagnostic sensitivity and specificity indexes of each included literature showed that the sensitivity and specificity indexes of radionuclide imaging in the diagnosis of thyroid nodules did not change significantly after the deletion of each included literature, which indicates that there is no significant publication bias and location bias in the included literature. The overall quality was stable and the results were relatively reliable.

\section{Discussion}

Thyroid tissue can specifically absorb radionuclides, and the size, number, and nature of thyroid nodules can be determined by ECT to display the distribution of radioactivity in the thyroid (18). Thyroid nodules are usually classified into hot, warm, cool, and cold nodules in radionuclide scans (19). Hot nodules are highly functional and are commonly seen in functional autonomous thyroid tumor adenoma. The radiographic imaging of the absorbed part of the nodules is significantly higher than that of the surrounding normal thyroid tissue (20). Warm nodules are more common in benign adenomas of the thyroid gland, nodular goiters, thyroid cysts, etc. The radiographic imaging of nodules is the same as that of the surrounding thyroid tissue. Cool nodules are more common in benign thyroid tumors, some of which also exist in malignant thyroid tumors. The radiographic distribution of these nodules is lower than that of surrounding thyroid tissue, showing sparse radiographic distribution. The radiometric distribution of cold nodules decreases significantly, and radiometric defects often appear. The difference between cold and cool nodules is the degree of reduction in iodine uptake at the nodular site (21). Several studies have suggested that radionuclide scanning (ECT) can help 
clinicians understand the functional status of the thyroid. Recently, it was reported that thyroid radionuclide imaging can effectively diagnose single or multiple thyroid nodules with decreased serum thyroid stimulating hormone (TSH). Moreover, it can be used to determine whether the thyroid nodules of patients are hot nodules with self-absorption function and clinical hyperthyroidism (22).

In order to further explore the overall effectiveness of radionuclide imaging in the diagnosis of thyroid nodules, this study screened and systematically meta-analyzed the relevant articles published so far. A total of 7 articles were included, involving a total of 2,629 patients. The results of the systematic evaluation and meta-analysis showed that the combined sensitivity of the diagnosis of thyroid nodules with radionuclide imaging was 0.66 , and the $95 \%$ CI range was $(0.46,0.89)$. The combined specificity was 0.36 , and its $95 \%$ CI was $(0.21,0.59)$; the combined positive LR was 0.66 , and its $95 \%$ CI was $(0.53,0.87)$; the combined negative LR was 0.67 , and its $95 \%$ CI was $(0.43,0.95)$; and the combined diagnostic OR was 4.45 , and its $95 \% \mathrm{CI}$ was $(0.25,10.57)$. The AUC for the diagnosis of thyroid nodules by radionuclide imaging was 0.38 . This shows that radionuclide imaging has a certain diagnostic value in the diagnosis of thyroid nodules, and its overall diagnostic sensitivity is good.

However, the AUC for the diagnosis of radionuclide imaging of thyroid nodules was relatively small, indicating that its diagnostic value was low. In the diagnosis of thyroid nodules, it was more biased towards the diagnosis of hot nodules and the differentiation of benign and malignant thyroid nodules. Such results are consistent with the research results of Klein Hesselink et al. [2016] (23). At the same time, studies have pointed out that the current diagnosis of thyroid nodules by radionuclide imaging is still controversial. Some researchers believe that although radionuclide scanning imaging has a good effect on the diagnosis of high-functioning thyroid nodules, and its detection is expensive and not suitable for large-scale clinical promotion. Another part of scholars pointed out that the specificity and accuracy of radionuclide scanning imaging for the diagnosis of thyroid nodules are poor, and it is of little help in clinical detection (24), which is consistent with the results of this study. Comparison on the included articles reveals that if the serum TSH of the thyroid nodule is significantly lower than the normal level, $99 \mathrm{mTc}$ radionuclide scanning imaging shows a very high diagnostic accuracy rate (25). This shows that when radionuclide scanning imaging is used to diagnose thyroid nodules, it is necessary to perform simultaneous detection with serum TSH levels to jointly improve the accuracy of diagnosis. Current studies have shown that radionuclide imaging of the thyroid gland has good diagnostic accuracy for many diseases caused by thyroid dysfunction, such as ectopic thyroid, destructive thyroiditis, and hyperthyroidism. This method is not effective for the diagnosis of hypofunctional and isofunctional thyroid nodules, but for patients with high-functional thyroid nodules, the iodine uptake method or the $99 \mathrm{~m}$ Tc uptake method However, in patients with high-functioning thyroid nodules, radionuclide ECT scan with iodine uptake or $99 \mathrm{mTc}$ uptake can provide a more accurate diagnosis of high-functioning thyroid nodules.

\section{Conclusions}

In this study, seven studies related to radionuclide scanning for thyroid nodule diagnosis were included for metaanalysis. The results showed that the sensitivity and specificity of thyroid nodules by radionuclide scanning imaging were 0.83 (95\% CI: 0.76, 0.89) and 0.36 (95\% CI: $0.21,0.59$ ), respectively, and the AUC was 0.72 . It was indicated that radionuclide scanning imaging had certain diagnostic value for thyroid nodules, but the overall diagnostic accuracy was not very high. The limitation of this study is that some relevant articles on clinical diagnosis of thyroid nodules using multiple methods may have been omitted during the literature selection process, resulting in a small sample size. In future research, it is necessary to further improve the retrieval method, optimize the extraction process of the key information in the included articles, and strive to find more relevant studies for comparative study, so as to enhance the comprehensive quality of the paper. In conclusion, this study provides some reference for exploring the application of radionuclide imaging in the clinical diagnosis of thyroid nodules.

\section{Acknowledgments}

Funding: None.

\section{Footnote}

Reporting Checklist: The authors have completed the PRISMA reporting checklist. Available at https://dx.doi. org/10.21037/gs-21-766

Conflicts of Interest: All authors have completed the ICMJE 
uniform disclosure form (available at https://dx.doi. org/10.21037/gs-21-766). The authors have no conflicts of interest to declare.

Ethical Statement: The authors are accountable for all aspects of the work in ensuring that questions related to the accuracy or integrity of any part of the work are appropriately investigated and resolved.

Open Access Statement: This is an Open Access article distributed in accordance with the Creative Commons Attribution-NonCommercial-NoDerivs 4.0 International License (CC BY-NC-ND 4.0), which permits the noncommercial replication and distribution of the article with the strict proviso that no changes or edits are made and the original work is properly cited (including links to both the formal publication through the relevant DOI and the license). See: https://creativecommons.org/licenses/by-nc-nd/4.0/.

\section{References}

1. Agrawal C, Guthrie L, Sturm MS, et al. Comparison of Thyroid Nodule Prevalence by Ultrasound in Childhood Cancer Survivors With and Without Thyroid Radiation Exposure. J Pediatr Hematol Oncol 2016;38:43-8.

2. Anil C, Kut A, Atesagaoglu B, et al. Metformin Decreases Thyroid Volume and Nodule Size in Subjects with Insulin Resistance: A Preliminary Study. Med Princ Pract 2016;25:233-6.

3. Lin M, Su Y, Huang Y, et al. Ultrasonographic evaluation of extracapsular vascular invasion for subcapsular nodules of the thyroid. Transl Cancer Res 2020;9:6464-70.

4. Demir C, Anil C, Bozkus Y, et al. Do Statins Affect Thyroid Volume and Nodule Size in Patients with Hyperlipidemia in a Region with Mild-to-Moderate Iodine Deficiency? A Prospective Study. Med Princ Pract 2018;27:1-7.

5. Lodewijk L, Vriens MR, Vorselaars WM, et al. Sameday fine-needle aspiration cytology diagnosis for thyroid nodules achieves rapid anxiety decrease and high diagnostic accuracy. Endocr Pract 2016;22:561-6.

6. Chianelli M, Bizzarri G, Todino V, et al. Laser ablation and 131-iodine: a 24-month pilot study of combined treatment for large toxic nodular goiter. J Clin Endocrinol Metab 2014;99:E1283-6.

7. Wharry LI, McCoy KL, Stang MT, et al. Thyroid nodules $(\geq 4 \mathrm{~cm})$ : can ultrasound and cytology reliably exclude cancer? World J Surg 2014;38:614-21.
8. Borysewicz-Sanczyk H, Dzieciol J, Sawicka B, et al. Practical Application of Elastography in the Diagnosis of Thyroid Nodules in Children and Adolescents. Horm Res Paediatr 2016;86:39-44.

9. Friedrich-Rust M, Vorlaender C, Dietrich CF, et al. Evaluation of Strain Elastography for Differentiation of Thyroid Nodules: Results of a Prospective DEGUM Multicenter Study. Ultraschall Med 2016;37:262-70.

10. Korkusuz H, Happel C, Heck K, et al. Percutaneous thermal microwave ablation of thyroid nodules. Preparation, feasibility, efficiency. Nuklearmedizin 2014;53:123-30.

11. Basharat R, Bukhari MH, Saeed S, et al. Comparison of fine needle aspiration cytology and thyroid scan in solitary thyroid nodule. Patholog Res Int 2011;2011:754041.

12. Deandreis D, Al Ghuzlan A, Auperin A, et al. Is (18) F-fluorodeoxyglucose-PET/CT useful for the presurgical characterization of thyroid nodules with indeterminate fine needle aspiration cytology? Thyroid 2012;22:165-72.

13. Lin JH, Chiang FY, Lee KW, et al. The role of neck ultrasonography in thyroid cancer. Am J Otolaryngol 2009;30:324-6.

14. Lumachi F, Varotto L, Borsato S, et al. Usefulness of $99 \mathrm{~m}$ Tc-pertechnetate scintigraphy and fineneedle aspiration cytology in patients with solitary thyroid nodules and thyroid cancer. Anticancer Res 2004;24:2531-4.

15. Muñoz Pérez N, Villar del Moral JM, Muros Fuentes MA, et al. Could 18F-FDG-PET/CT avoid unnecessary thyroidectomies in patients with cytological diagnosis of follicular neoplasm? Langenbecks Arch Surg 2013;398:709-16.

16. Piccardo A, Puntoni M, Treglia G, et al. Thyroid nodules with indeterminate cytology: prospective comparison between 18F-FDG-PET/CT, multiparametric neck ultrasonography, 99mTc-MIBI scintigraphy and histology. Eur J Endocrinol 2016;174:693-703.

17. Riazi A, Kalantarhormozi M, Nabipour I, et al.

Technetium-99m methoxyisobutylisonitrile scintigraphy in the assessment of cold thyroid nodules: is it time to change the approach to the management of cold thyroid nodules? Nucl Med Commun 2014;35:51-7.

18. Poller DN, Megadmi H, Ward MJA, et al. Hürthle Cells on Fine-Needle Aspiration Cytology Are Important for Risk Assessment of Focally PET/CT FDG Avid Thyroid Nodules. Cancers (Basel) 2020;12:3544.

19. Wang C, Zhang X, Yang X, et al. PET response assessment in apatinib-treated radioactive iodine-refractory thyroid 
cancer. Endocr Relat Cancer 2018;25:653-63.

20. Granchi S, Vannacci E, Biagi E. Characterization of benign thyroid nodules with HyperSPACE (Hyper Spectral Analysis for Characterization in Echography) before and after percutaneous laser ablation: a pilot study. Med Ultrason 2017;19:172-8.

21. Gabler AS, Kühnel C, Winkens T, et al. Assessment of Minimum 124I Activity Required in Uptake Measurements Before Radioiodine Therapy for Benign Thyroid Diseases. J Nucl Med 2016;57:1201-6.

22. Yamaga LYI, Cunha ML, Campos Neto GC, et al. 68GaDOTATATE PET/CT in recurrent medullary thyroid carcinoma: a lesion-by-lesion comparison with 111Inoctreotide SPECT/CT and conventional imaging. Eur J Nucl Med Mol Imaging 2017;44:1695-701.

23. Klein Hesselink EN, Brouwers AH, de Jong JR, et al. Effects of Radioiodine Treatment on Salivary

Cite this article as: Song X, Luo Z, Sun H, Hao L. Systematic review and meta-analysis of the diagnostic value of radionuclide imaging for thyroid nodules. Gland Surg 2021;10(12):33513361. doi: $10.21037 / \mathrm{gs}-21-766$
Gland Function in Patients with Differentiated Thyroid Carcinoma: A Prospective Study. J Nucl Med 2016;57:1685-91.

24. Vrachimis A, Stegger L, Wenning C, et al. [(68)Ga] DOTATATE PET/MRI and [(18)F]FDG PET/CT are complementary and superior to diffusion-weighted MR imaging for radioactive-iodine-refractory differentiated thyroid cancer. Eur J Nucl Med Mol Imaging 2016;43:1765-72.

25. Giovanella L, Campenni A, Treglia G, et al. Molecular imaging with (99m)Tc-MIBI and molecular testing for mutations in differentiating benign from malignant follicular neoplasm: a prospective comparison. Eur J Nucl Med Mol Imaging 2016;43:1018-26.

(English Language Editor: A. Kassem) 